\title{
Central nervous system penetration and efficacy of targeted therapies used in non-small cell lung cancer and melanoma with brain metastases
}

\author{
Sidra Khalid*1, Aariez Khalid ${ }^{2}$, Abdo Haddad ${ }^{3}$, Timothy Spiro ${ }^{3}$, Hamed Daw ${ }^{3}$ \\ ${ }^{1}$ Internal Medicine, Fairview Hospital, Cleveland Clinic, United States \\ ${ }^{2}$ Biomedical Science, University of Guelph, Canada \\ ${ }^{3}$ Department of Hematology and Oncology, Fairview Hospital, Cleveland Clinic, United States
}

Received: March 10, 2018

Accepted: April 15, 2018

Online Published: May 3, 2018

DOI: $10.5430 /$ jst.v8n2p13

URL: http://dx.doi.org/10.5430/jst.v8n2p13

\begin{abstract}
Recently, many targeted therapies have been approved for treating brain metastases in non-small cell lung cancer and melanoma patients. In this article, the targeted therapies and their mechanism of action will be reviewed. It will highlight the central nervous system penetration of the targeted therapies. The article will also relate the efficacy of these drugs as seen in clinical trials, which would help guide clinicians when managing these patients.
\end{abstract}

Key Words: Non-small cell lung cancer, Melanoma, EGFR inhibitor, ALK inhibitor, BRAF inhibitor, MEK inhibitor, VEGF inhibitor, Immunotherapy

\section{INTRODUCTION}

Currently, the FDA has approved many targeted therapies for non-small cell lung cancer and melanoma associated with brain metastases. These targeted therapies inhibit specific molecules such as EGFR, ALK, VEGF, BRAF, and MEK. Additionally, immunotherapy is also being utilized for treating brain metastases. These drugs provide a novel way to treat brain metastases, which has historically been treated with local surgery, stereotactic radiosurgery, or whole brain radiation therapy. ${ }^{[1]}$ Since these treatments have side effects and delay systemic therapy, the treatment with the newer targeted therapies is ideal. This article will focus on the targeted therapies, their mechanism of action, central nervous system (CNS) penetration and efficacy.

\section{NON-SMALL CELL LUNG CANCER (NSCLC)}

The targeted therapies which are used for advanced NSCLC act on the epidermal growth factor receptor (EGFR), anaplastic lymphoma kinase (ALK) receptors and vascular endothelial growth factor (VEGF).

\subsection{EGRF inhibitors \\ 2.1.1 Erlotinib}

Erlotinib is an EGFR inhibitor and is used as a first line treatment for NSCLC. It competes against ATP to reversibly bind to the catalytic domain of the EGFR. This blocks signal transduction events and potential tumorigenic effects. ${ }^{[2]}$ Erlotinib and its active metabolite OSI-420's CNS penetration was assessed in four patients with NSCLC. They were States.

Published by Sciedu Press 
given $150 \mathrm{mg}$ of erlotinib. The results showed that erlotinib CSF concentration was $5.1 \%$ and OSI-420 CSF concentration was $5.8 \%$. Therefore, erlotinib maybe a good treatment option for NSCLC with brain metastases. ${ }^{[3]}$ Additionally, Togashi et al. stated that erlotinib's CSF concentration increased with its plasma concentration, which could be useful in cases of refractory CNS metastases in NSCLC patients. This suggests that erlotinib administered at a higher dose than $150 \mathrm{mg}$, could be beneficial. ${ }^{[4]}$ In a retrospective study by Bai et al., 40 patients with advanced NSCLC were given erlotinib. In the patients with intracranial disease, $4(10 \%)$ had partial response, 21 (52.5\%) had stable disease, and 15 (37.5\%) had progressive disease. The patients with EGFR mutations had a disease control rate of $80 \%$. The study showed that erlotinib is moderately active in NSCLC with brain metastases and is more effective in EGFR mutation positive individuals. ${ }^{[5]}$

\subsubsection{Gefitinib}

Gefitinib is an EGFR inhibitor and is used for NSCLC. ${ }^{[6]}$ Gefitinib and its CNS penetration was assessed in 15 Japanese patients diagnosed with NSCLC and brain metastases. They were administered $250 \mathrm{mg}$ of gefitinib and $150 \mathrm{mg}$ of erlotinib. The CSF concentration and penetration rate were higher for erlotinib than gefitinib and this led to higher CNS response rates in the erlotinib patients. ${ }^{[7]} \mathrm{Sim}-$ ilarly, Zhao et al. conducted a study in which 22 patients with lung adenocarcinoma and brain metastases were given gefitinib. They showed that the CSF concentration of gefitinib was low, and correlated with the plasma concentration. Hence, to achieve higher CSF concentration in NSCLC with brain metastases, higher doses of gefitinib should be used. ${ }^{[8]}$

A prospective trial was undertaken by Ceresoli et al. in which gefitinib was given to NSCLC patients with brain metastases to assess its safety and efficacy. In the 41 patients, $250 \mathrm{mg}$ of gefitinib was administered. $4(10 \%)$ had a partial response, $7(17 \%)$ had a stable disease and the overall disease control rate was $27 \%$. The conclusion was that gefitinib can be active in brain metastases in NSCLC. The standard therapy, prior chemotherapy and whole brain radiation therapy (WBRT), was also assessed and revealed disappointing results. As a result, the study concluded that gefitinib is a new treatment option. ${ }^{[9]}$ Similarly, a meta-analysis of 7 trials looked at erlotinib/gefitinib with WBRT, WBRT alone, or WBRT with chemotherapy. It concluded that erlotinib/gefitinib plus WBRT was superior to WBRT alone, as the overall response rate was 2.16. ${ }^{[10]}$ Additionally, gefitinib's effect was studied in NSCLC patients with brain metastases. Of the 57 patients, 14 had brain metastases, 1 had complete response, 5 had partial responses, and 8 had stable disease in the brain. This study found that in refractory NSCLC, gefitinib has efficacy and is well tolerated. ${ }^{[11]}$

\subsubsection{Afatinib}

Afatinib is an EGFR inhibitor used for metastatic NSCLC with EGFR exon 19 deletions or exon 21 substitution mutations. ${ }^{[12]}$ Tamiya et al. studied afatinib's CSF penetration rate. The study included 11 patients with EGFR positive advanced NSCLC, who were given afatinib at $40 \mathrm{mg} / \mathrm{day}$. On day 8 , the CSF penetration rate was $2.45+/-2.91 \%$. The overall response rate was $27.3 \%$. This study concluded that afatinib was effective for NSCLC with uncommon EGFR mutations. ${ }^{[13]}$ In the two trials, LUX-Lung 3 and LUX-Lung 6, NSCLC with EGFR mutations and brain metastases patients treated with afatinib had improved progression free survival (8.2 vs. 5.4 months) and overall response rate (73\% vs. $25 \%)$ when compared to standard platinum therapy. ${ }^{[14]}$

\subsubsection{Osimertinib}

Osimertinib is an EGFR inhibitor that is selective for EGFRTKI sensitizing and T790M resistance mutations in NSCLC. Soria et al. conducted a study which included 556 patients with previously untreated EGRF mutation-positive (exon 19 deletion or L858R) locally advanced or metastatic NSCLC. They were distributed into three treatment groups. Patients received either $80 \mathrm{mg}$ of osimertinib, or $250 \mathrm{mg}$ of gefitinib, or $150 \mathrm{mg}$ of erlotinib. CNS progression occurred in $17(6 \%)$ patients on osimertinib and $42(15 \%)$ patients who were given the standard EGFR-TKI therapy. All these results stated that osimertinib had a prolonged progression free survival than the current standard EGFR mutation-positive treatment. $^{[15]}$

\subsection{ALK inhibitors}

\subsubsection{Crizotinib}

Crizotinib is FDA approved for metastatic NSCLC ALK+ mutations. It binds to ATP in a competitive manner, which results in binding and inhibition of ALK kinase and ALK fusion proteins. It also blocks the c-met pathway. These then cause inhibition of tumor growth. Crizotinib's CSF concentration has been studied by Costa et al. and Metro et al. Their results show that crizotinib has poor CFS concentration, as evidenced by low CSF to serum ratios. However, despite the poor CSF concentration some patients with brain metastases benefited from crizotinib. This finding requires further study to determine the underlying mechanisms. Crizotinib is also a p-glycoprotein substrate. The efficacy of crizotinib was studied in the PROFILE 1014 trial, in which crizotinib was compared to standard chemotherapy (platinum doublets). The results showed that intracranial disease control rate was $56 \%$ vs. $25 \%$ respectively. ${ }^{[16]}$

\subsubsection{Ceritinib}

Certinib is used for metastatic NSCLC ALK+ mutations. It is a tyrosine kinase, Cytochrome P450 3A, and Cytochrome 
P450 2C9 inhibitor. It binds and blocks ALK kinase, ALK fusion proteins and ALK point mutation variants, and inhibits cell growth in ALK-overexpressing tumor cells. A preclinical rat model demonstrated ceritinib's penetration of the blood brain barrier with a CSF and plasma ratio of $15 \%$. In the ASCEND 3 trial, ceritinib was administered to 50 patients with brain metastases at baseline, 27 of whom had prior radiotherapy. In 17 patients, the intracranial response rate was $58.9 \%$ and disease control rate was $82.4 \%$. This study suggests that ceritinib can be active in brain metastases, especially in those who have not had any prior treatment with ALK inhibitors. ${ }^{[16]}$

\subsubsection{Alectinib}

Alectinib is an ALK inhibitor which is used for NSCLC with ALK+ mutations. Alectinib has a potent antineoplastic activity against intracranial lesions due to its high rate of CNS penetration. It is not a substrate for p-glycoprotein and hence is not effluxed. The efficacy of alectinib was assessed in several trials. In the AF-002JG study, of 21 patients with brain metastases, $6(29 \%)$ had complete response, 5 $(24 \%)$ had partial response, $8(38 \%)$ had stable disease and $2(10 \%)$ had progressive disease. Similarly, the NP28761 trail showed a response rate of $68.8 \%$ and disease control rate was $100 \%$ in patients with baseline intracranial lesions treated with alectinib. ${ }^{[16]}$

\subsection{VEGF inhibitor \\ Bevacizumab}

Bevacizumab is a recombinant human neutralizing VEGF IgG antibody, which inhibits tumor angiogenesis. It is FDA approved for NSCLC. Bevacizumab acts on intracranial lesions, not by disrupting the blood brain barrier, but through neutralizing the VEGF within the lumen of capillaries. The efficacy of bevacizumab was assessed in the BRAIN study. In the trial, NSCLC patients with asymptomatic brain metastases were divided into two arms: Arm \#1 - bevacizumab, paclitaxel, and carboplatin; Arm \#2 - bevacizumab and erlotinib. The response rates for the intracranial and extracranial metastases in both arms were identical. Bevacizumab had an acceptable safety profile in this patient population. ${ }^{[17]}$

\section{Melanoma}

Targeted therapies that are used in melanoma act on the mitogen-activated protein (MAP) kinase pathway. In this pathway, RAS protein is activated, which then stimulates the RAF kinases ARAF, BRAF and RAF1. These kinases then cause MEK kinase phosphorylation that activates ERK kinase. This controls cyclin D1, which causes cellular division and progression. ${ }^{[18]}$ The targeted therapies available for the treatment of melanoma are BRAF inhibitors, MEK inhibitors, and immunotherapy.

Published by Sciedu Press

\subsection{BRAF inhibitors}

\subsubsection{Vemurafenib}

Vemurafenib is a BRAF inhibitor which is used for unresectable or metastatic melanoma with BRAF (V600) mutations. According to Mittapalli et al. vemurafenib is effluxed by p-glycoprotein and BCRP, which constitute the blood brain barrier. Vemurafenib's CNS distribution is severely restricted. ${ }^{[19]}$ However, in a phase 2 clinical trial by McArthur et al., 146 metastatic melanoma patients were given vemurafenib. They were distributed in two cohorts, (1) previously untreated brain metastases and (2) previously treated brain metastases. In cohort 1 , the intracranial best overall response rate was $18 \%$. The study authors concluded that vemurafenib use in melanoma with brain metastases has a clinically meaningful response rate. ${ }^{[20]}$ In another study by Dummer et al. vemurafenib was used for BRAF (V600) mutation-positive melanoma patients with symptomatic brain metastases. For intracranial and extracranial sites, an overall partial response was reached in 10 out of 24 patients (42\%). Out of the 19 patients with measurable intracranial disease, 7 (37\%) had intracranial tumor regression, and $3(16 \%)$ had a partial response. $^{[21]}$

\subsubsection{Dabrafenib}

Dabrafenib is a BRAF protein inhibitor that decreases cellular proliferation of tumor cells containing a mutated BRAF gene. In a phase 1 dose escalation trial, patients with melanoma and untreated brain metastases were included. 9 out of 10 had reductions in the size of their brain lesions. ${ }^{[22]}$ Even with the response seen in this trial, the CNS distribution of dabrafenib was uncertain. Mittapalli et al. looked at the CNS penetration of dabrafenib. The results showed that dabrafenib is a substrate for p-glycoprotein and BCRP, which efflux dabrafenib. Hence, dabrafenib has limited CNS penetration. However, the study also compared the CNS distribution of vemurafenib to dabrafenib and found that dabrafenib had greater CNS penetration at a similar dose. ${ }^{[23]}$ Thus, dabrafenib appears to be a better option than vemurafenib. In a study by Rodgers et al. the CNS penetration of dabrafenib was evaluated. They concluded that there were quantifiable concentrations of dabrafenib in the plasma, but CSF had low concentrations. ${ }^{[24]}$

In the BREAK-MB, multicenter phase 2 trial, dabrafenib's safety and efficacy were assessed in 172 patients with BRAF V600E, V600K mutations and metastatic melanoma with at least one measurable brain metastasis. They were divided into two cohorts, (A) no local therapy and (B) with progression after local therapy. The results showed that the overall intracranial objective response in cohort A was 39\% and in cohort $\mathrm{B}$ was $31 \%$. As BRAF inhibitors have a high rate of intracranial objective response, they should be considered in BRAF mutated melanoma. ${ }^{[1]}$ 
Table 1. CNS distribution and Efficacy of Targeted Therapies in Advanced NSCLC

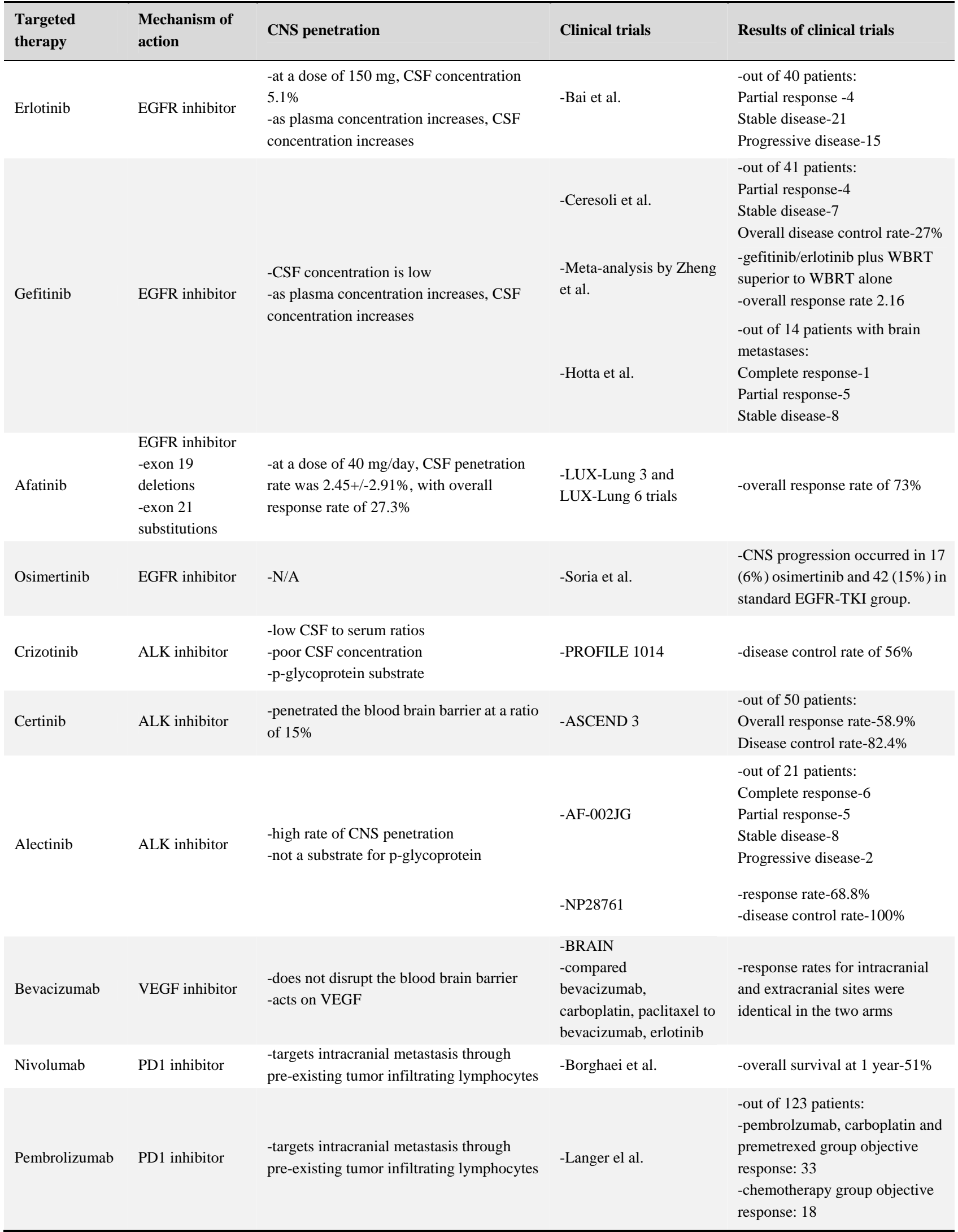


Table 2. CNS distribution and Efficacy of Targeted Therapies in Advanced Melanoma

\begin{tabular}{|c|c|c|c|c|}
\hline $\begin{array}{l}\text { Targeted } \\
\text { therapy }\end{array}$ & $\begin{array}{l}\text { Mechanism of } \\
\text { action }\end{array}$ & CNS penetration & Clinical trials & Results of clinical trials \\
\hline Vemurafenib & BRAF inhibitor & $\begin{array}{l}\text {-effluxed by p-glycoprotein } \\
\text { and BCRP } \\
\text {-CNS distribution severely } \\
\text { restricted }\end{array}$ & -McArthur et al. & $\begin{array}{l}\text {-intracranial best overall response rate } \\
18 \% \text { in patients who had untreated } \\
\text { brain metastases } \\
\text {-out of } 19 \text { patients with measurable } \\
\text { intracranial lesions, } 7 \text { had tumor } \\
\text { regression, } 3 \text { partial response }\end{array}$ \\
\hline Dabrafenib & BRAF inhibitor & $\begin{array}{l}\text {-effluxed by p-glycoprotein } \\
\text { and BCRP } \\
\text {-darafebnib greater } \\
\text { penetration into the brain } \\
\text { than vemurafenib at a } \\
\text { similar dose }\end{array}$ & $\begin{array}{l}\text {-BREAK-MB, two cohorts } \\
\text { Cohort A: no local therapy } \\
\text { Cohort B: progression after } \\
\text { local therapy }\end{array}$ & $\begin{array}{l}\text {-overall intracranial objective response: } \\
\text { Cohort A: } 39 \% \\
\text { Cohort B: } 31 \%\end{array}$ \\
\hline Trametinib & MEK inhibitor & $\begin{array}{l}\text {-restricted brain distribution } \\
\text {-substrate of p-glycoprotein } \\
\text { and BCRP }\end{array}$ & $\begin{array}{l}\text {-Robert et al. } \\
\text {-darafenib and trametinib } \\
\text { compared to vemurafenib } \\
\text { alone }\end{array}$ & $\begin{array}{l}\text {-improved overall survival in the } \\
\text { combined group } \\
\text {-objective response rate: } \\
\text { Dabrafenib and trametinib: } 64 \% \\
\text { Vemurafenib: } 51 \%\end{array}$ \\
\hline Ipilimumab & $\begin{array}{l}\text { Monoclonal antibody } \\
\text { against CTLA4 }\end{array}$ & $\begin{array}{l}\text {-acts against brain } \\
\text { metastases through } \\
\text { activated } \mathrm{T} \text { cells }\end{array}$ & $\begin{array}{l}\text {-Margolin et al. } \\
\text { Cohort A: no steroids, } \\
\text { asymptomatic } \\
\text { Cohort B: steroids and } \\
\text { symptomatic } \\
\text {-Hodi et al. }\end{array}$ & $\begin{array}{l}\text {-ipilimumab used in both cohorts: } \\
\text { Disease control in cohort A - } 12 \\
\text { Disease control in cohort B - } 2 \\
\text {-ipilimumab with gp100 vaccine had } \\
\text { improved overall survival (10.1 } \\
\text { months) }\end{array}$ \\
\hline Nivolumab & PD1 inhibitor & $\begin{array}{l}\text {-targets intracranial } \\
\text { metastasis through } \\
\text { pre-existing tumor } \\
\text { infiltrating lymphocytes }\end{array}$ & -Cohen et al. & $\begin{array}{l}\text {-groups with nivolumab showed } \\
\text { statistically significant progression free } \\
\text { survival than ipilimumab } \\
\text {-nivolumab has overall survival at } 1 \\
\text { year of } 51 \% \text { compared with docetaxel } \\
\text { at } 39 \%\end{array}$ \\
\hline Pembrolizumab & PD1 inhibitor & $\begin{array}{l}\text {-targets intracranial } \\
\text { metastasis through } \\
\text { pre-existing tumor } \\
\text { infiltrating lymphocytes }\end{array}$ & -Robert et al. & $\begin{array}{l}\text {-pembrolizumab prolonged progression } \\
\text { free survival and overall survival than } \\
\text { ipilimubmab }\end{array}$ \\
\hline
\end{tabular}

\subsection{MEK inhibitor}

\section{Trametinib}

Trametinib is a MEK inhibitor used with dabrafenib for unresectable or metastatic melanoma with BRAF (V600E or $\mathrm{V} 600 \mathrm{~K})$ mutations. As there is emerging resistance encountered downstream to BRAF in the MAPK signaling pathway, trametinib offers a novel way to overcome this resistance by acting through MEK-1/2. Hence, concurrent therapy with a BRAF inhibitor and trametinib is preferred. In a phase 3 trial in which trametinib was combined with dabrafenib, there was improved overall survival in untreated metastatic melanoma with BRAF V600E or V600K mutations. ${ }^{[25]}$ Trametinib's CNS penetration was evaluated by Vaidhyanathan et al. in which they concluded that trametinib has a restricted brain distribution as it is a p-glycoprotein and BCRP substrate. ${ }^{[26]}$

Published by Sciedu Press

\subsection{Immunotherapy}

Ipilimumab is a humanized monoclonal anti-CTLA4 (cytotoxic T lymphocyte antigen-4) used for the treatment of unresectable or metastatic melanoma. ${ }^{[1]}$ Ipilimumab does not have a good CNS penetration because of its molecular size. It acts against the brain metastases through activated T-cells. ${ }^{[27]}$ In a phase III trial, ipilimumab, with or without a gp100 peptide vaccine, compared to gp100 peptide alone, showed an improved overall survival (10.1 months) for previously treated metastatic melanoma. ${ }^{[28]}$ In a phase 2 trial, 72 patients with melanoma and brain metastases were divided into two cohorts, A - neurologically asymptomatic with no corticosteroids, B - symptomatic with corticosteroids. Ipilimumab was administered in both cohorts. Disease control in the brain was shown in $12(24 \%)$ in cohort A and $2(10 \%)$ in cohort B $(10 \%)$. The trial authors concluded that ipili- 
mumab had some activity in advanced melanoma with brain metastases. $^{[27]}$

\section{Melanoma AND NSCLC}

\subsection{PD1/PDL1 inhibitors}

In the human body, the peptide fragments on the antigen presenting cells are recognized by the T cells, which in turn activate cellular immunity. This T cell stimulation is usually deactivated by a pathway that consists of programmed cell death receptor 1 (PD-1). When PD1 binds with PDL1, which is on tumor cells, it downregulates the T cells ability to mount a response against tumor cells. PD1 and PDL1 antibodies, such as nivolumab and pembrolizumab, restore the antitumor response in patients with melanoma. ${ }^{[29]}$ It is hypothesized that there might be tumor infiltrating lymphocytes, and immune modulation by these agents allows cytotoxic $\mathrm{T}$ cells migration into the brain. ${ }^{[30]}$

\subsubsection{Nivolumab}

Nivolumab is used for metastatic melanoma and chemotherapy resistant metastatic NSCLC. In a multicenter, randomized trial, 945 patients with prior untreated, unresectable, or metastatic melanoma were divided into three groups; nivolumab with ipilimumab, nivolumab, and ipilimumab. The study concluded that progression free survival was statistically significant in the nivolumab groups than ipilimumab alone. ${ }^{[31]}$ Nivolumab is FDA approved as a monotherapy or in combination with ipilimumab in unresectable or metastatic melanoma. In a phase 3 trial, nivolumab was compared to docetaxel in advanced NSCLC patents. The overall survival at 1 year with nivolumab was $51 \%$ and for docetaxel was $39 \%$. $^{[32]}$

\subsubsection{Pembrolizumab}

Pembrolizumab is FDA approved for unresectable or metastatic melanoma. A phase 3 trial showed that pembrolizumab demonstrated a longer progression free survival and overall survival compared to ipilimumab. ${ }^{[33]}$ Pembrolizumab is also approved as a first line agent for metastatic NSCLC with expression of PDL1 on at least $50 \%$ of tumor cells, and as second line agent for metastatic NSCLC after platinum based chemotherapy is ineffective in controlling progression. Based on a phase 2 clinical trial of 123 patients, carboplatin and pemetrexed pembrolizumab with/without in advanced NSCLC concluded that an objective response was achieved in 33 with pembrolizumab and 18 with chemotherapy. The study concluded that pembrolizumab, carboplatin and pemetrexed combination is effective and well tolerated. ${ }^{[34]}$

\section{Conclusion}

The various targeted therapies reviewed in this paper have shown activity against brain metastases in NSCLC and melanoma patients (see Tables 1 and 2). Most of the targeted therapies have a low CNS penetration, however in clinical trials they have proven to be clinically effective. The targeted therapies provide newer treatment options for an advanced stage disease, when compared to surgery, stereotactic radiotherapy or chemotherapy. By utilizing the information about the CNS distribution and efficacy of the targeted therapies, clinicians could tailor a management plan for patients with metastatic NSCLC and melanoma. To further our understanding about the efficacy of targeted therapies, clinical trials should be conducted to evaluate targeted therapies as first-line treatment options for patients with NSCLC and melanoma with brain metastases. Through specific research about the targeted therapies, we would be able to highlight their effectiveness as well as their associated adverse effects and outcomes, which would aid in the decision-making process.

\section{CONFlicts OF INTEREST Disclosure}

The authors declare that there is no conflict of interest statement.

\section{REFERENCES}

[1] Ramanujam S, Schadendorf D, Long GV. Systemic therapies for melanoma brain metastases: which drug for whom and when? Chin Clin Oncol. 205 Jun. 4.

[2] PubChem, Open Chemistry Database. Erlotinib. Available from: https://pubchem.ncbi.nlm.nih.gov/compound/176870 Accessed 2017.

[3] Togashi Y, Masago K, Fukado M, et al. Cerebrospinal fluid concentration of erlotinib and its active metabolite OSI-420 in patients with central nervous system metastases of non-small cell lung cancer. J Thorac Oncol. 2010 Jul; 5: 950-955. https://doi.org/10.109 7/JT0.0b013e3181e2138b

[4] Togashi Y, Masago K, Fukudo M, et al. Efficacy of increased-dose erlotinib for central nervous system metastases in non-small cell lung cancer patients with epidermal growth factor receptor mutation. Cancer Chemother Pharmacol. 2011 Oct; 68: 1089-1092. https://doi.org/10.1007/s00280-011-1691-z.

[5] Bai H, Han B. The effectiveness of erlotinib against brain metastases in non-small lung cancer patients. Am J Clin Oncol. 2013 Apr; 36: 110-115. PMid:22391431. https://doi.org/10.1097/COC.0b $013 e 3182438 c 91$

[6] PubChem, Open Chemistry Database. Gefitinib. Available from: https://pubchem.ncbi.nlm.nih.gov/compound/1236 31\#section=Top. Accessed 2017. 
[7] Togashi Y, Masago K, Masuda S, et al. Cerebrospinal fluid concentration of gefitinib and erlotinib in patients with non-small cell lung cancer. Cancer Chemother Pharmacol. 2012 Sep; 70: 399-405. https://doi.org/10.1007/s00280-012-1929-4

[8] Zhao J, Chen M, Zhong W, et al. Cerebrospinal fluid concentration of gefitinib in patients with lung adenocarcinoma. Clin Lung Cancer. 2013 Mar; 14: 188-193. https ://doi.org/10.1016/j.cllc.2 012.06 .004

[9] Ceresoli GL, Cappuzzo F, Gregorc V, et al. Gefitinib in patients with brain metastases from non-small-cell lung cancer: a prospective trial. Ann Oncol. 2004 Jul; 15: 1042-1047. https ://doi .org/10. 109 3/annonc/mdh276

[10] Zheng MH, Sun HT, Xu JG, et al. Combining whole-brain radiotherapy with gefitinib/erlotinib for brain metastases from nonsmall-cell lung cancer: a meta-analysis. Biomed Res Int. 2016 Feb. https://doi.org/10.1155/2016/5807346

[11] Hotta K, Kiura K, Ueoka H, et al. Effect of gefitinib ('Iressa', ZD1839) on brain metastases in patients with advanced non-smallcell lung cancer. Lung Cancer. 2004 Nov; 46: 255-261. https: //doi.org/10.1016/j.lungcan.2004.04.036

[12] PubChem, Open Chemistry Database. Afatinib. Available from: https://pubchem.ncbi.nlm.nih.gov/compound/10184653. Accessed 2017.

[13] Tamiya A, Tamiya M, Nishihara T, et al. Cerebrospinal fluid penetration rate and efficacy of afatinib in patients with EGFR mutationpositive non-small cell lung cancer with leptomeningeal carcinomatosis: a multicenter prospective study. Anticancer Res. 2017 Aug; 37: 4177-4182. https://doi.org/10.21873/anticanres.11806

[14] Wynes MW, Wolf J. IASLC. Afatinib shows clinical benfit for lung cancer patients with brain metastasis. Available from: https: //www.iaslc.org/articles/afatinib-shows-clinica l-benefit-lung-cancer-patients-brain-metastases. Accessed 2017.

[15] Soria JC, Ohe Y, Vansteenkiste J, et al. Osimertinib in untreated EGFR-mutated advanced non-small cell lung cancer. N Engl J Med. 2018 Jan; 378: 113-125. https ://doi .org/10.1056/NEJMoa17 13137

[16] Toyokawa G, Seto T, Takenoyama M, et al. Insights into brain metastasis in patients with ALK+ ling cancer: is the brain truly a sanctuary? Cancer Metastasis Rev. 2015 Sep; 34: 797-805. https://doi .org/10.1007/s10555-015-9592-y

[17] Kim YH, Nagai H, Ozasa $H$, et al. Therapeutic strategy for non-smallcell lung cancer pateints with brain metastases (Review). Biomed Rep. 2013 Sep; 1: 691-696.

[18] Welbrock C, Hurlstone A. BRAF as therapeutic target in melanoma. Biochem Pharmacol. 2010 Sep; 80: 561-567. PMid:20350535. https://doi.org/10.1016/j.bcp.2010.03.019

[19] Mittapalli RK, Vaidhyanathan S, Sane R, et al. Impact of pglycoprotein (ABCB1) and breast cancer resistance protein (ABCG2) on the brain distribution of a novel BRAF inhibitor: vemurafenib (PLX4032). J Pharmacol Exp Ther. 2012 Jul; 342: 33-40. https: //doi.org/10.1124/jpet.112.192195

[20] McArthur GA, Maio M, Arance A, et al. Vemurafenib in metastatic melanoma patients with brain metastases: an open-label, singlearm, phase 2, multicenter study. Ann Oncol. 2017; 28: 634-641. https://doi.org/10.1093/annonc/mdw641
[21] Dummer R, Goldinger SM, Turtschi CP, et al. Vemurafenib in patients with BRAF (V600) mutation-positive melanoma with symptomatic brain metastases: final results of an open-label pilot study. Eur $\mathbf{J}$ Cancer. 2014 Feb; 50: 611-621. https://doi.org/10.1016/j. ejca.2013.11.002

[22] Falchook GS, Long GV, Kurzrock R, et al. Dabrafenib in patients with melanoma, untreated brain metastases, and other solid tumours: a phase 1 dose-escalation trial. Lancet. 2012 May; 370: 1893-1901. https://doi.org/10.1016/S0140-6736(12)60398-5

[23] Mittapalli RK, Vaidhyanathan S, Dudek AZ, et al. Mechanisms limiting distribution of the threonine-protein kinase B-RAF V600E inhibitor dabrafenib to the brain: implications for the treatment of melanoma brain metastases. J Pharmacol Exp Ther. 2013 Mar; 344: 655-664. https://doi.org/10.1124/jpet.112.201475

[24] Rodgers L, McCully CML, Gross A, et al. TRTH-33. Comparative plasma and cerebrospinal fluid pharmacokinetics of BRAF and MEK inhibitors in a nonhuman primate model. Neuro Oncol. 2017 May. 19: iv59. https://doi.org/10.1093/neuonc/nox083.243

[25] Robert C, Karaszewska B, Schachter J, et al. Improved overall survival in melanoma with combined dabrafenib and trametinib. $\mathrm{N}$ Engl J Med. 2015 Jan; 372: 30-39. https://doi .org/10.1056/NEJM oa1412690

[26] Vaidhyanathan S, Mittapalli RK, Sarkaria JN, et al. Factors influencing the cns distribution of a novel MEK-1/2 inhibitor: implications for combination therapy for melanoma brain metastases. Drug Metab Dispos. 2014 Aug; 42: 1292-1300. https://doi.org/10.1124/ dmd.114.058339

[27] Margolin K, Ernstoff MS, Hamid O, et al. Ipilimumab in patients with melanoma and brain metastases: an open-label, phase 2 trial. Lancet. 2012 May; 13: 459-465. https://doi .org/10.1016/S1 470-2045 (12) 70090-6

[28] Hodi FS, O'Day SJ, McDermott DF, et al. Improved survival with ipilimumab in patients with metastatic melanoma. N Engl J Med. 2010 Aug; 19: 711-723. https://doi.org/10.1056/NEJMoa1003466

[29] Brahmer JR, Tykodi SS, Chow LQ, et al. Safety and activity of antiPD-L1 antibody in patients with advanced cancer. N Engl J Med. 2012 Jun; 366: 2455-2465. https://doi .org/10.1056/NEJMoa 1200694

[30] Cohen JV, Kluger HM. Systemic immunotherapy for the treatment of brain metastases. Front Oncol. 2016 Mar; 6: 49. PMid:27014624. https://doi.org/10.3389/fonc. 2016.00049

[31] Wolchok JD, Kluger H, Callahan MK, et al. Nivolumab plus ipilimumab in advanced melanoma. N Engl J Med. 2013 Jul; 369: 122-133. https://doi.org/10.1056/NEJMoa1302369

[32] Borghaei H, Paz-Ares L, Hom L, et al. Nivolumab versus docetaxel in advanced nonsquamous non-small cell lung cancer. $\mathrm{N}$ Engl $\mathrm{J}$ Med. 2015 Oct. 373: 1627-1639. https ://doi .org/10.1056/NEJMoa 1507643

[33] Robert C, Schachter J, Long GV, et al. Pembrolizumab versus ipilimumab in advanced melanoma. N Engl J Med. 2015 Jun; 372: 2521-2532. https://doi.org/10.1056/NEJMoa1503093

[34] National Cancer Institute. FDA Expands approval of pembrolizumab for first-line treatment of non-small cell lung cancer. Available from: https://www. cancer.gov/news-events/cancer-cur rents-blog/2017/fda-pembrolizumab-lung-expanded. Accessed 2017. 